\title{
Implementation and Analysis of Density based Location Aware Routing in Intermittently Connected MANETs
}

\author{
Anushruti Soni \\ PG Scholar: CSE Department \\ Inderprastha Engineering College \\ Ghaziabad, U.P., India
}

\author{
Chandani Saxena \\ Associate Professor: CSE Department \\ Inderprastha Engineering College \\ Ghaziabad, U.P., India
}

\begin{abstract}
Mobile Ad-Hoc Networks (MANETS)[7] are an emerging technology that allows establishing instant communication infrastructures for civilian and military applications. An Intermittently Connected Mobile Ad hoc Networks (ICMANET)[4] operates in environments where the nodes do not form a completely connected network. Existing geographical routing protocol like location-aware routing for delay-tolerant networks (LAROD) [1,3] enhanced with a location service has shown to work well in IC-MANET. But the challenge here is to design a routing protocol to handle intermittently connectivity and dense/sparse topologies and also the performance of this geographical routing protocol degrades with varying network node densities such as sparse and dense topologies. For sparse systems, distribution of location information will probably be very slow. For dense systems, the transfer of location data may start to consume too much bandwidth locally at the dense areas. In this work, we propose a density based geographical routing algorithm called density based location-aware routing for delay tolerant networks (DLAROD). D-LAROD calculates the density of the network based on location of the neighboring nodes information that counter for every route update that is performed at the time of route discovery process. The D-LAROD scheme is compared with the existing LAROD protocol[1] and is shown to have a competitive edge, both in terms of delivery ratio and overhead.
\end{abstract}

\section{Keywords}

Node Density, Intermittent connectivity, Location aware routing protocol, Ad-Hoc on demand routing protocol (AODV)

\section{INTRODUCTION}

Most Routing Protocols of Mobile Ad-Hoc Networks (MANETs)[5] were studied using open space models where nodes were able to move throughout the entire simulation area. However, a more realistic topology should account for restricted mobility of nodes, such as in an urban city setting where there may be concentrations of nodes within specific areas (such as within buildings) and low density of nodes in other areas (such as in parks and roadways). Consequently, the simulation area should be partitioned into smaller subareas with varying densities to model such areas, called dense/ sparse areas. Routing the packets in fully connected Mobile Ad-Hoc Networks (MANETs) has been studied to great extent, but the assumption on full connectivity is generally not valid in a real system. The challenge here is to design a routing protocol to handle intermittently connectivity and dense/sparse topologies. This study characterized the effect of dense/sparse topologies on MANET routing performance and proposes an extension for an existing LAROD-LoDiS[2], routing protocol to work in such topologies called D-LAROD. The proposed protocol improved the performance of MANETs by reducing the communication overhead incurred during the routing processes by implementing a new density based geographical routing algorithm. The proposed routing algorithm was based on the density and connectivity of the nodes and not just the number nodes.

Advantages of the proposed algorithm: 1.D-LAROD calculates the density of the network based on which the dissemination of location data takes place. 2. The D-LAROD scheme is compared with the existing LAROD protocol and is shown to have a competitive edge, both in terms of delivery ratio and overhead.

Modules used in the proposed algorithm: 1. Network Configuration-We consider networks with 35 nodes. The mobile nodes are randomly and uniformly deployed in a square area of size $1200 \times 900 \mathrm{~m}$. The node transmission range is set to $250 \mathrm{~m}$. Nodes move at the speed of $5 \mathrm{~m} / \mathrm{ms}$ across in the network field. Data traffic is generated for 100 packets per millisecond over the whole network[6]. Each packet is randomly and uniformly assigned to a source, excluding nodes that are one hop from the destination. The chosen source queues the assigned packets and transmits them as soon as possible. The maximum queue length per node is set to 50 packets. A newly generated packet is accepted by the source only if its buffer is not full.

Neighbor Discovery 


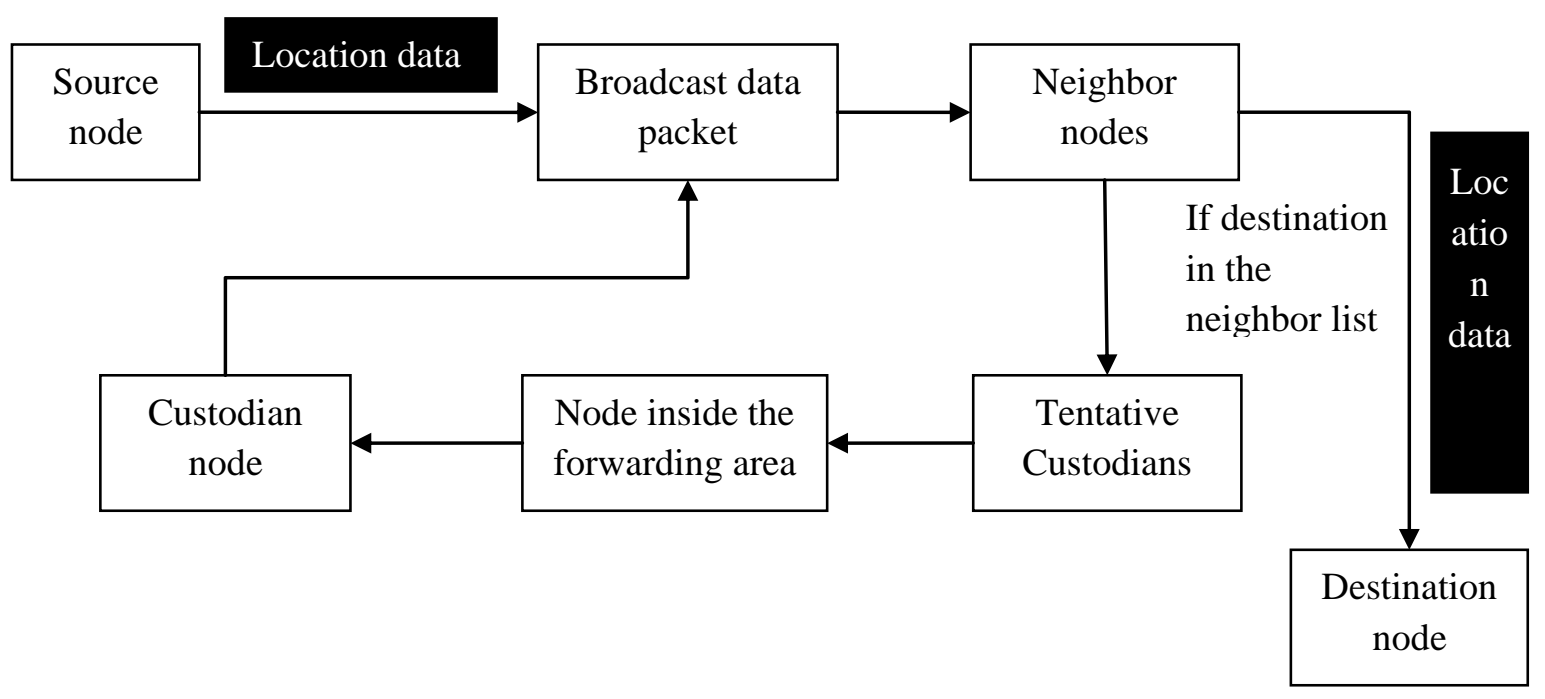

In MANET Communications, all the nodes are dynamic. So, the neighbor list of each node gets updated according to the node movement. The nodes go out of the communication range or come inside the communication range of other node with respect to the direction of movement. The list of nodes present inside the communication range[4] of a particular node is called as the neighbor list of that node. The distance between all the nodes has been calculated. Based on the distance, each and every node shortlisted the nodes which are present at the distance less than its communication range. In our simulation, $250 \mathrm{~m}$ is the communication range[4].

\section{Custodian Selection}

A custodian forwards a message toward the destination by simply broadcasting it. All nodes within a predefined forwarding area[6] are called tentative custodians and are eligible to forward the packet. All tentative custodians set a delay timer td specific for each node, and the node whose delay timer expires first is selected as the new custodian. The new custodian forwards the message in the same manner as the previous custodian. The old custodian that forwarded the message and other tentative custodians will overhear this broadcast and conclude that a new node has taken over custody of the packet. If the current custodian does not overhear any such broadcast within an interval of $\operatorname{tr}($ rebroadcast time)[10], it repeats the broadcast of the message until a new custodian becomes available due to node mobility.

\section{Data Transmission}

The data packet generated at the source node is transmitted to the destination node via the custodian nodes. When a packet has been received by the destination, it sends an acknowledgement packet (ack)[8] to stop further transmission of a packet by custodians and tentative custodians. All nodes that hear an acknowledgement will store the acknowledgement information until the packet times out. If a node receives a packet for which it previously has received an acknowledgement, then it broadcasts an acknowledgement packet to stop further transmission of the packet.

\section{Performance evaluation}

All mobile nodes are randomly scattered with a uniform distribution. Randomly select one of the deployed nodes as the source node. We evaluate our proposed method with respect to the following metrics: Packet delivery ratio[1,8], E2E latency[1,5], Packet loss ratio[1,9].

Packet delivery ratio: is the ratio of the number of report messages the sink receives to the total number of report messages the source node sends.

Packet loss ratio: measures the ratio of packets have been dropped during transmission time

End to end latency: It refers to the time taken for a packet to be transmitted across a network from source to destination.

These parameter values are recorded in the trace file during the simulation by using record procedure. The recorded details are stored in the trace file. The trace file is executed by using the Xgraph to get graph as the output.

\section{LITERATURE SURVEY}

In this section we give an overview of relevant research in the MANET and DTN area regarding geographical routing and location services. And also we discussed different criteria that the routing in mobile Ad-Hoc networks and density parameters, which has been studied extensively and routing protocols like AODV, DSR and GPSR have been suggested. All these protocols assume that there exists a contemporaneous path between sender and receiver. In networks without contemporaneous paths, but where node mobility can overcome partitions, a new type of routing algorithm is required. We have discussed existing protocols like LAROD-LoDiS protocol and mobility models and location services which do not support for different density areas. Mobile Ad-Hoc Networks (MANETs) are an emerging technology that allows establishing instant communication infrastructures for civilian and military applications. An AdHoc network is self-organizing and communicates mostly through multi hop wireless links. Mobility of network members, limited resources (e.g., bandwidth and energy supply) and potentially large number of mobile nodes make routing in Ad-Hoc networks extremely challenging. Node mobility has been acknowledged as one of the key challenges of Ad-Hoc networking with direct impact on protocol performance. Early models, such as Random Walk, Random Direction and Random Waypoint, spray and wait consider nodes moving on a free-space planar surface and deploying MANET in urbanized areas with a mixture of vehicular and pedestrian traffic and disaster areas would fit into the scenario where node densities vary from time to time.

Calculation of Neighboring nodes density- Network physical density is considered as dense when large number nodes are in close proximity of one another within a particular area and vice versa for sparse. However, when determining the 
network density, one should also consider the connectivity of the network in terms of transmission range that covers the particular area. Thus, in this study, network density is based on the number of nodes found in a particular area and the connectivity of the nodes. The density of a network is defined based on the transmission range of the nodes as given below:

- The number of neighbors surrounding a node is denoted by its degree $d$

- A node with degree $\mathrm{d}=0$ is said to be isolated from the rest of the network

- dmin denotes the minimum degree of nodes and is considered as the smallest degree of all nodes in the network

- A network is said to be connected if there exists a path between every pair of nodes, otherwise it is disconnected

- A connected network always has a minimum degree $\mathrm{dmin}>0$ but the reverse implication is not true

- A network is k-connected if for each pair of node exists $\mathrm{k}$ mutually independent paths connecting them

Density calculation: The density is calculated based on (1)(3).

$\mathrm{P}(\mathrm{k}-\mathrm{con}) \approx(1-\mathrm{e}-\mu) \mathrm{n} \quad(1)$

$\mu=\rho \times \pi \times(\mathrm{r} 0 * \mathrm{r} 0)$

$\rho=n / A$

where

$\mathrm{P}=$ Probability of the connectivity

$\mathrm{n}=$ Neighbor count

$\mathrm{A}=$ Pre-defined area size

$\rho=$ density

$\pi=3.142$

$\mathrm{r} 0 * \mathrm{r} 0=$ Transmission radius

In this study the value of $\mathrm{k}$ is set to1. This means that in any particular network mentioned as dense given the probability of the connection of $\mathrm{P}(\mathrm{k}$-con $) \geq 0.95$ where $\mathrm{k}=1$, there is 1 mutually independent path connecting the nodes in the particular network area. Thus the network is categorized as (almost surely) 1-connected. This also implies that for any neighbors found within the transmission range of a particular node they are at most 2 hops away from each other. The node density of the network areas in this study will be based on the formulae provided for $\mathrm{P}(\mathrm{k}-\mathrm{con})$. Therefore an area is considered dense when a MANET source node identifies that:

- It neighbors are at most 2 hops away from it and it has a mutually exclusive path to other neighboring nodes that is independent of one another $\mathrm{P}(1$-con $) \geq$ 0.95 Sparse areas will be areas where nodes are isolated from a network or from one another.

- Nodes in a sparse neighbor cannot guarantee at least a single connection in the network $(\mathrm{P}(1-\mathrm{con}) \leq 0.95)$

- The minimal neighbor node degree for sparse areas could be dmin $=0$. Thus, the node could be disconnected from the network.
Proposed routing algorithm - In this section we present DLAROD routing algorithm that is used in the study. DLAROD is a geographical routing protocol for IC-MANETs that use greedy packet forwarding when possible. When greedy forwarding is not possible, the node that currently holds the packet waits until node mobility makes it possible to resume greedy forwarding.

The node forwards a message toward the destination by simply broadcasting it between the neighboring nodes. All nodes within a predefined forwarding area are called tentative nodes and are eligible to forward the packet to the destination with the help of neighboring nodes. All tentative nodes set a delay timer td specific for each node, and the node whose delay timer expires first is selected as the new node. The new node forwards the message in the same manner as the previous node. The old node that forwarded the message and other tentative nodes will overhear this broadcast and conclude that a new node has taken over custody of the packet. If the current node does not overhear any such broadcast within an interval of $\operatorname{tr}$ (rebroadcast time), it repeats the broadcast of the message until a new node becomes available due to node mobility.

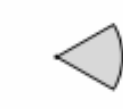

Sector (a) Reuleaux (b)

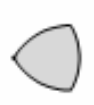

\section{Fig.2 D-LAROD forwarding area}

When the time to live tTTL for a packet, which is expressed as duration, expires, a packet is deleted by its custodian. This is done to prevent a packet from indefinitely trying to find a path to its destination. The forwarding area can have many shapes as shown in fig2 Examples of shapes include a $60^{\circ}$ circle sector, a Reuleaux, triangle, or a circle [Fig. 2(a)-(c)]. The longest distance between two points within these shapes must be the assumed radio range. If we want to maximize the probability of determining a new custodian, then the forwarding area should include all nodes that guarantee progress toward the destination [Fig. 2(d)]. In this paper, we have chosen progress forwarding area. When a packet has been received by the destination, it sends an acknowledgement packet (ack) to stop further transmission of a packet by custodians and tentative custodians. All nodes that hear an acknowledgement will store the acknowledgement information until the packet times out. If a node receives a packet for which it previously has received an acknowledgement, then it broadcasts an acknowledgement packet to stop further transmission of the packet. Fig. 3 shows the pseudo code for D-LAROD routing protocol.

Source node at data packet generation
Initiating route discovery when
Access source node \& intermediate neighbor node cache
If neighbor !=null
Calculate neighbor count as NC
Return NC
Calculate density of the NC by the formula




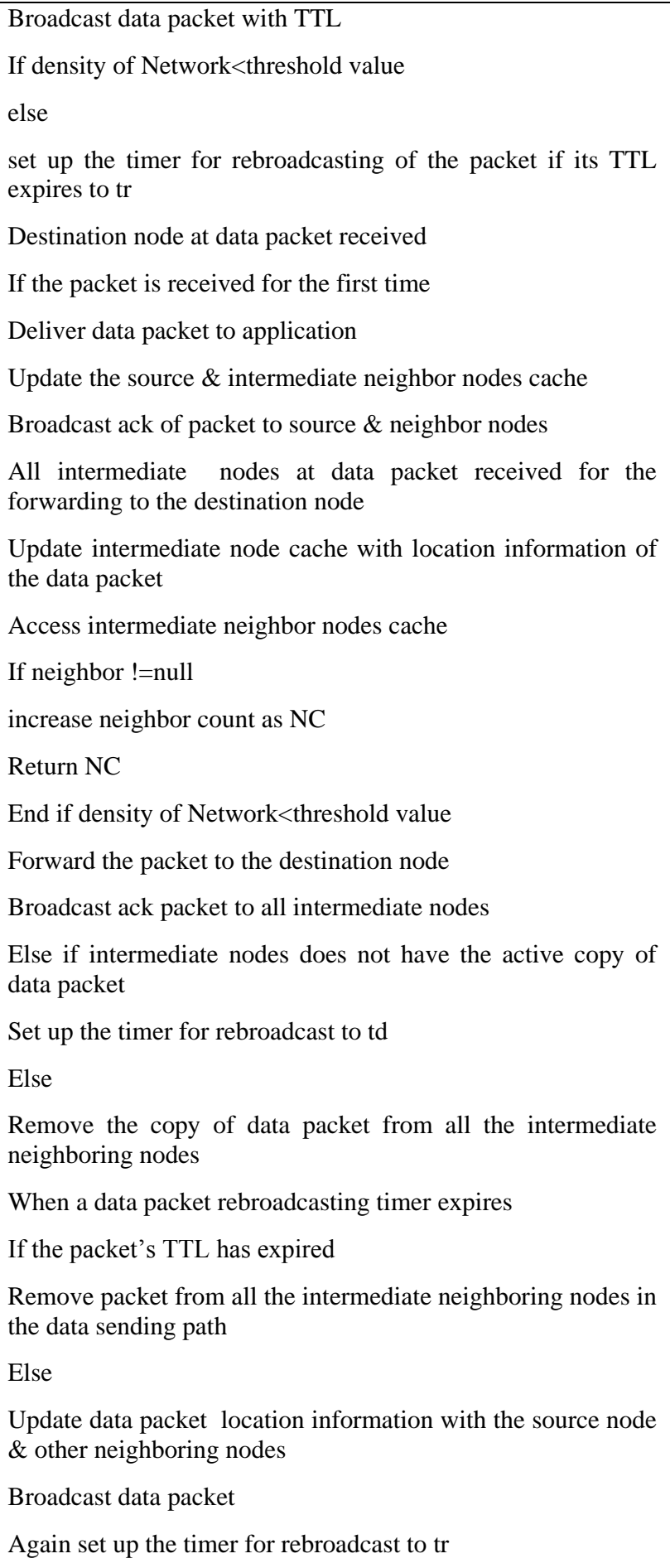

Fig3- pseudo code for D-LAROD

\section{EVALUATION}

In this section, we have shown the results of our evaluations of D-LAROD. The routing protocols have been evaluated in the network simulator ns-2. Delivery ratio and effort required for each generated data packets are the two main evaluation metrics used.

The delivery ratio determines the quality of service as perceived by the user or application and it is the most important evaluation criterion. The effort will be measured as the number of transmissions performed per generated data packet. Figure 4 shows the xgraph of packet delivery ratio.

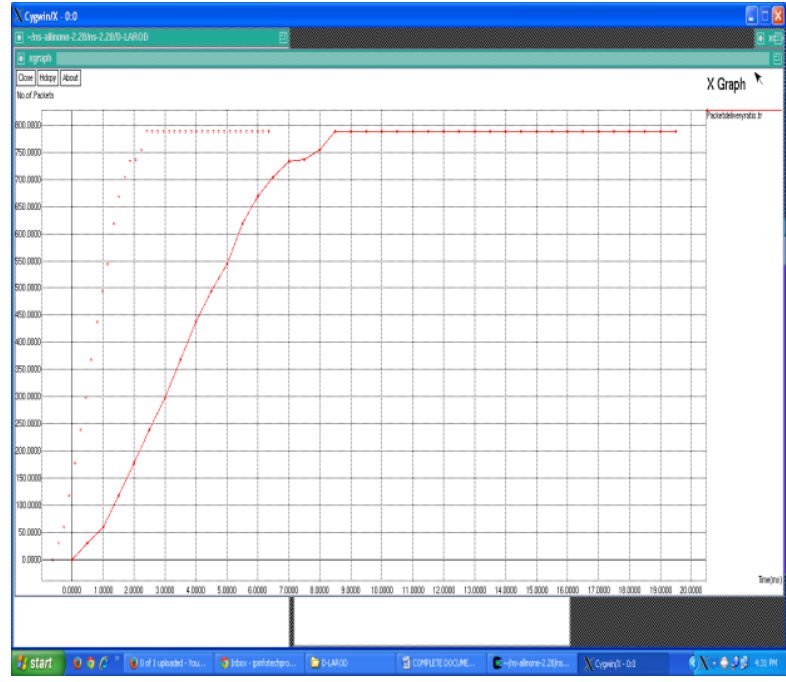

Fig4 packet life time Vs packet delivery ratio

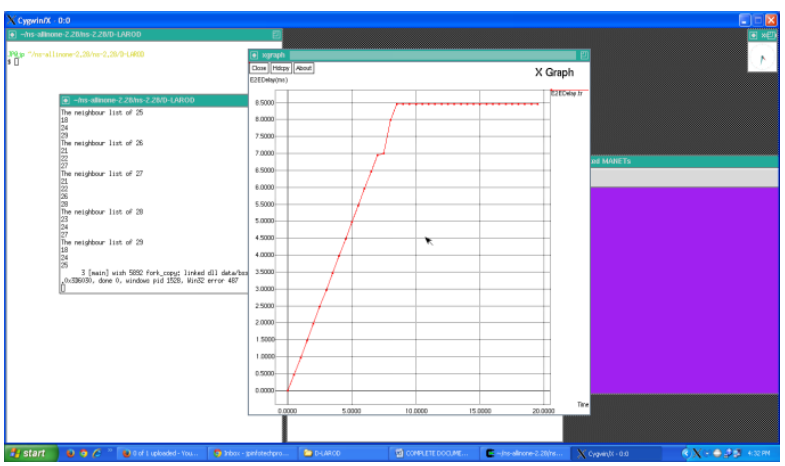

Fig5 packet life time Vs packet loss ratio

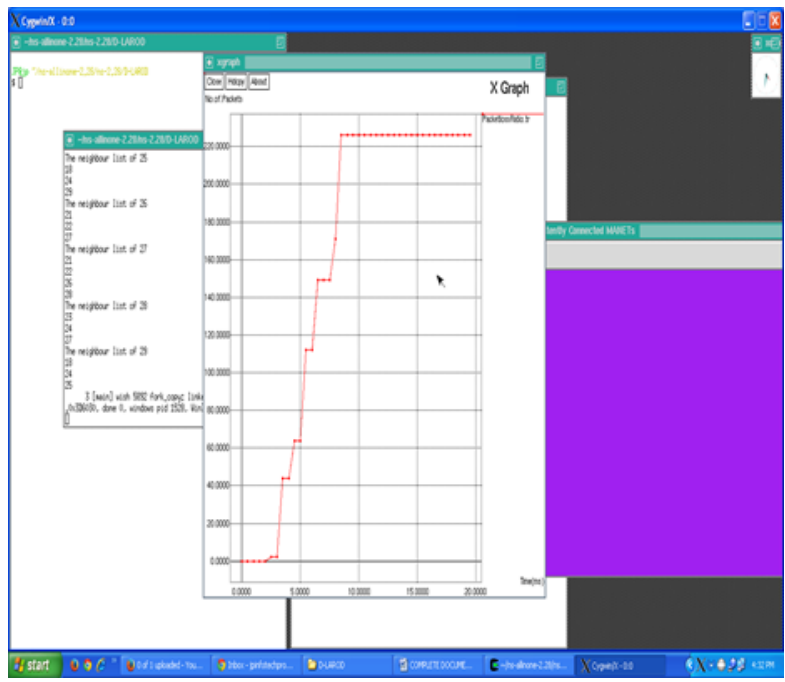

Fig 6 packet life time Vs E2Edelay

Figure 5 shows the Xgraph of packet loss ratio. When the packet is transmitted between the nodes the packet loss is enquired sometimes due to congestion in network, TTL expires etc. Figure 6 shows the end to end delay graph. When the packet is transferred from source to destination the overall time is calculated taken by packet from source to reach its destination. 


\section{CONCLUSION}

In this paper we identified a new class of dense and highly mobile networks not well addressed by conventional DTN or MANET approaches. We proposed a new Density based algorithm to the LAROD protocol that broadcasts the packets based on the density of the network. If it is low density network (sparse) normal broadcasts otherwise less broadcasts in case of dense network. It improved the performance of a simple DTN protocol. Simulations of our implementation in a dense and highly mobile network show significant performance improvements over regular Spray-and- Wait and LAROD. Further studies can be done on very large systems where the challenge will be how the location information is distributed for all the nodes in the system. To do this approach, some kind of data compression or approximation methods for nodes located far away can be employed.

\section{REFERENCES}

[1] E. Kuiper and S. Nadjm-Tehrani, "Geographical routing in intermittently connected ad hoc networks," in Proc. 1st IEEE Int.Workshop Opportunistic Netw., 2008, pp. $1690-1695$.

[2] T. Spyropoulos, K. Psounis, and C. S. Raghavendra, "Spray and wait: An efficient routing scheme for intermittently connected mobile networks," in Proc. ACM SIGCOMM Workshop Delay-Tolerant Netw., 2005, pp. 252-259.

[3] T. Camp, J. Boleng, and L. Wilcox, "Location information services in mobile ad hoc networks," in
Proc. IEEE Int. Conf. Commun., 2002, pp. 3318-3324.

[4] R. R. Choudhury, "Brownian gossip: Exploiting node mobility to diffuse information in ad hoc networks," in Proc. Int. Conf. Collaborative Comput.: Netw., Appl. Worksharing, 2005, pp. 1-5.

[5] F. Warthman, Delay-Tolerant Networks (DTNs): A Tutorial v1.1. Warthman Assoc., Mar. 2003. [Online] Available:http://www.dtnrg.org/docs/tutorials/warthman 1.1.df

[6] S. M. Das, H. Pucha, and Y. C. Hu, "Performance comparison of scalable location services for geographic ad hoc routing," in Proc. IEEE 24th Annu.Joint Conf. IEEE Comput.Commun.Soc., 2005, pp. 1228- 1239.

[7] V. Cerf, S. Burleigh, A. Hooke, L. Torgerson, R. Durst, K. Scott, K. Fall, and H.Weiss, Delay-tolerant networking architecture. RFC 4838.[Online]. Available: ftp://ftp.rfc-editor.org/in-notes/rfc4838.txt.

[8] R. Bruno and M. Nurchis, "Survey on diversity-based routing in wireless mesh networks: Challenges and solutions," Comput. Commun., vol. 33, no. 3, pp. 269282, Feb. 2010.

[9] C.E. Perkins, E.M. Royer,“Ad-hoc On-Demand Distance Vector Routing”, WMCSA-1999, IEEE.

[10] Abolhasan, M., T. Wysocki, E., Dutkiewicz, (2004),“A review of routing protocols for mobile Ad-Hoc networks", Ad-Hoc Networks, 2: pp. 1-22. 